\title{
Incentive mechanisms for promoting D2D communications in cellular networks
}

\section{Bhed Bahadur Bista*, Jiahong Wang and Toyoo Takata}

Faculty of Software and Information Science,

Iwate Prefectural University,

Takizawa City, Iwate Ken 020-0693, Japan

Email: bbb@iwate-pu.ac.jp

Email:wjh@iwate-pu.ac.jp

Email: takata@iwate-pu.ac.jp

*Corresponding author

\begin{abstract}
Recently, device-to-device (D2D) communications have been proposed for efficient utilisation of resources of cellular networks by offloading some traffic of base station (BS) to direct links between user equipments (UEs), i.e., cellular users. While there are many works on optimisation of resources sharing in D2D communications in cellular networks, a little works has been done to promote D2D communications and attract users to adopt and take part in it. In this paper, in order to encourage users to adopt D2D communications, we present users achievable data rate both in D2D link and cellular link when they ask base station (BS) for contents. If the data rate in D2D link is less than cellular link, users will ask for reward for taking part in D2D communications. We also present how much reward will be awarded for taking part in D2D communications. By simulation, we show the percentage of users asking for rewards and the amount of reward they ask for taking part in D2D communications under various scenarios. The simulation results show that all users do not ask reward and may wilfully take part in D2D communications without reward.
\end{abstract}

Keywords: D2D communications; LTE; cellular networks; SINR; achievable data rate; reward.

Reference to this paper should be made as follows: Bista, B.B., Wang, J. and Takata, T. (2018) 'Incentive mechanisms for promoting D2D communications in cellular networks', Int. J. Space-Based and Situated Computing, Vol. 8, No. 1, pp.1-8.

Biographical notes: Bhed Bahadur Bista received his BEng in Electronics from the University of York, England and MS and PhD in Information Science from the Tohoku University, Japan. After his PhD, he worked at the Miyagi University, Japan, for one year as a Research Associate and moved to the Iwate Prefectural University, also in Japan as an Assistant Professor. Currently, he is an Associate Professor with the Faculty of Software and Information Science at the same university. His research interests include energy efficient networks, mobile networks, sensor networks, ad hoc networks, cognitive radio networks, and cellular networks. He has organised international workshops and has actively taken part as a Program Chair, Track Chair and a program committee member in various international conferences including flagship IEEE AINA, $N B i S, B W C C A$ and IMIS. He is a member of IPSJ, IEICE and IEEE.

Jiahong Wang received his BE from the Northeast University, China, and ME from the Shenyang Institute of Computing Technology, the Chinese Academy of Sciences, China, both in computer science. He received his $\mathrm{PhD}$ from the University of Tsukuba, Japan. Now, he is a Professor at the Faculty of Software and Information Science, Iwate Prefectural University, Japan. His research interests include sensor network, concurrency control, transaction processing, distributed/parallel processing, data mining, and modelling and performance evaluation. $\mathrm{He}$ is a member of IEEE, IEICE, and IPSJ.

Toyoo Takata received his $\mathrm{PhD}$ in Information and Computer Sciences from the Osaka University, Japan, in 1989. In 1989, he was a Research Associate at the Department of Information and Computer Sciences, Osaka University. In 1993, he was an Associate Professor at the Graduate School of Information Science, Nara Institute of Science and Technology. Since 1998, he has been a Professor at the Faculty of Software and Information Science, Iwate Prefectural University. He is a member of IEEE, ACM, IEICE, IPSJ and SITA.

This paper is a revised and expanded version of a paper entitled 'Mechanism for adopting device-to-device communication in cellular networks' presented at 20th International Conference on Network-Based Information Systems, Ryerson University, Toronto Canada, 24-26 August 2017. 


\section{Introduction}

The most widely used high performance mobile wireless devices in the world are smartphones and tablets, whose number is increasing year by year. The proliferation of these devices and constant use of them to access the internet have strained the wireless access points specially cellular network access points. Almost all these devices are frequently used to upload and download videos, photos, audio files or access SNS services generating a huge amount of data traffic which is increasing year by year (Cisco, 2017). In order to satisfy ever increasing demand for wireless access and huge traffic flow, wireless cellular networks are also evolving and increasing their network capacity by integrating new technologies and shifting their paradigms. Currently, the cellular network is in fourth generation (4G) which is replacing $3 \mathrm{G}$ around the world. The $4 \mathrm{G}$ is being actively developed and is well-known by 3GPP LTE-Advanced (3GPP, n.d.; Ghosh et al., 2010). Besides providing higher channel capacity by introducing multi-antenna technology and carrier aggregation, LTE-Advanced is shifting its paradigm of communication model known as device-to-device (D2D) communication by taking advantages of high performance wireless devices (Asadi et al., 2014; Doppler et al., 2009). Unlike complex technologies involved in accommodating high demand of users, D2D can significantly improve resource utilisation of wireless cellular networks by letting a pair of $\mathrm{D} 2 \mathrm{D}$ devices which are in proximity of each other to communicate directly instead of communicating via base station (BS) [know as evolved node (eNB) base station in LTE].

In D2D communication, two devices called user equipments (UEs), which are in proximity of each other, can establish a direct communication link using the licensed band. Because of their proximity, there is limited interference from other users even if they use the same licensed band. Since D2D communication can be established by sharing channel, the eNB can provide service to other UEs using the same channel thereby increasing the throughput of the network. Moreover, since D2D pair communication range is assumed to be shorter than UEs to eNB, UEs will reduce the energy consumption significantly also (Datsika et al., 2016).

The main focus of the recent works on D2D communication is how spectrum/channel can be efficiently shared between D2D pairs and cellular user equipments (cUEs) in cellular networks so that there is minimum interference and maximum throughput in the network (Lin et al., 2014; Zulhasnine et al., 2010). Some works also focus on energy efficiency of D2D devices also (Ansari et al., 2016; Datsika et al., 2016). All the works assume that the BS as the central controller determines which devices will have to take part in D2D communication for maximisation of throughput and efficient use of spectrum. However, in practice, cellular users pay for their data plan. Moreover, there will be more delay in link establishment in D2D communication than with the BS. Users may not be willing to take part in D2D communication if they do not find the benefit or do not receive reward for doing so. It is essential that incentive mechanism for promoting D2D communications should be available to attract users to take part in D2D communications in order to offload cellular network traffics.

In this paper, we investigate how D2D pairs will benefit by taking part in D2D communications. When users know that the achievable data rate is higher in D2D link than cellular link with BS, they may be encouraged to take part in D2D communications without rewards. In our proposal, when a new cellular user requests content from BS, the BS first finds if there is a content provider within the D2D communication range. We assume that the BS has some mechanism of finding the content provider within its coverage area and how it does is beyond the scope of this paper. If it finds a content provider, it calculates the achievable data rate in D2D link and cellular link for the user and sends the information including the content requester's and the content provider's locations so that the requesting user can calculate and confirm the information it has received from the BS. User will then decide if it will take part in D2D communication without reward or with reward.

The paper is organised as follows. In Section 2, we present the related works. In Section 3, we present the system model. In Section 4, we show simulation results and analysis of our proposal followed by conclusions in Section 5 .

\section{Related work}

Many works in D2D communications mainly focus on interference management and resource allocations (Xiao et al., 2014; Yu et al., 2011; Ye et al., 2014). There are very few works on D2D communications in which users are encouraged to take part or are provided incentives to take part in D2D communications.

In $\mathrm{Li}$ and Guo (2015), authors propose two types of incentive mechanism for users to join D2D communication based on two market models - open market and closed market - in which the network operator and users are players who are interested to maximise their profits only. In open market model, authors use Stackelberg game-based incentive mechanism with a fixed total budget announced by the operator to reward D2D users who will have to compete with each other by switching to D2D communication mode. In case of closed market, authors have proposed an auction-based incentive mechanism in which each potential D2D pair asks for a payment from the network operator for switching to D2D communication mode. The network operator evaluates the prices asked by users, and determines whom to pay the reward for the network resources it gains from users switching to D2D communication mode.

In Zhang et al. (2015) and Chen et al. (2017), authors propose contract-theoretic model in which the BS hires the UEs as employees to fulfil the content transmission task using D2D communication. The BS as an employer offers contracts to UEs that specify different performance-reward 
combination for different UEs preferences. The UEs, as employees, select contracts that are best fit to their own preferences. The UEs with higher performance will obtain higher rewards.

The above approaches are all reward-based approach in which UEs are assumed to take part in D2D communications only if there is reward available. However, in order to promote D2D communications, it is essential to inform UEs that they may benefit more if they take part in D2D communications than cellular communications, in terms of achievable data transmission rates.

\section{System model}

In our system model, we consider only one cell of a cellular network with one BS at the centre of the cell. Within the coverage area of the BS, there are several cUE which are served by the BS and D2D user equipment (dUE) pairs in which one is a content requester/receiver and the other is a content provider/sender. When the content receiver UE requests the $\mathrm{BS}$ for content, the $\mathrm{BS}$ will directly serve the receiver if the content provider is not in the proximity of the receiver. If the content provider is within the $\mathrm{D} 2 \mathrm{D}$ pair forming range, the $\mathrm{BS}$ will provide an opportunity to form a D2D pair and the receiver can receive content directly from the content provider in D2D link. It is assumed that the content provider is always ready to establish D2D communication whenever it is possible or allowed to do so by the BS. When the D2D pair can be formed, the receiver may wilfully agree to receive content in D2D link because it is advantageous for it to do so. If not the receiver will ask for rewards from the BS for receiving content in D2D link instead of receiving it in cellular link from the BS. The receiver makes the decision based on the achievable data rates in D2D link and cellular link.

Figure 1 Interference when receiving content in D2D link

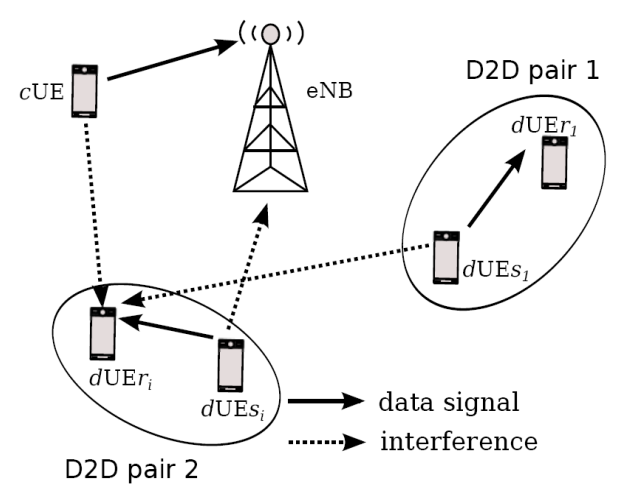

\subsection{Data transmission rate}

Whether a receiver wilfully agrees to take part in D2D communication or ask for reward depends upon the achievable data transmission rate. When the receiver is taking part in D2D communication, we consider only uplink case since resource sharing in this case affects only the BS, which can easily mitigate it by coordination. Figure 1 shows interference to the receiver when it receives content in D2D link. In the figure, the D2D receiver $\left(d U E r_{i}\right)$ in $\mathrm{D} 2 \mathrm{D}$ pair 2 is interfered by cUE and $d U E s_{1}$ in $\mathrm{D} 2 \mathrm{D}$ pair 1 as it shares the channel with them.

The data transmission rate is related to the signal to interference plus noise ratio (SINR). The SINR at receiver $r_{i}$ in D2D pair 2 in Figure 1 is as shown in equation (1).

$$
\operatorname{SINR}\left(r_{i}\right)=\frac{P\left(s_{i}\right) G\left(s_{i}, r_{i}\right)}{N+I+\operatorname{Inf}}
$$

where

$$
\begin{aligned}
\text { Inf } & =\sum_{c U E_{k}} P\left(c U E_{k}\right) G\left(c U E_{k}, r_{i}\right) \\
& +\sum_{j \neq i} P\left(s_{j}\right) G\left(s_{j}, r_{i}\right)
\end{aligned}
$$

where

$\left(s_{i}, r_{i}\right) \quad$ is a D2D pair in which $s_{i}$ is a sender and $r_{i}$ is a receiver.

$P\left(s_{i}\right) \quad$ is transmit power of $s_{i}$.

$G\left(s_{i}, r_{i}\right) \quad$ is channel gain between $\left(s_{i}, r_{i}\right)$.

$P\left(s_{j}\right) \quad$ is transmit power of sender $s_{j}$, of another D2D pair, to receiver $r_{j}$.

$G\left(s_{j}, r_{i}\right) \quad$ is channel gain between $\left(s_{j}, r_{i}\right)$.

$P\left(c U E_{k}\right) \quad$ is transmit power of cellular $c U E_{k}$ to $e N B$.

$G\left(c U E_{k}, r_{i}\right)$ is channel gain between cellular $c U E_{k}$ and D2D receiver $r_{i}$.

$N \quad$ is additive white Gaussian noise.

I is inter-cell interference.

The channel gain or link gain can be modelled in terms of distance between the sender and the receiver as shown below.

$$
\begin{aligned}
& G\left(s_{i}, r_{i}\right)=d\left(s_{i}, r_{i}\right)^{-\alpha} \\
& G\left(s_{j}, r_{i}\right)=d\left(s_{j}, r_{i}\right)^{-\alpha} \\
& G\left(c U E_{k}, r_{i}\right)=d\left(c U E_{k}, r_{i}\right)^{-\alpha}
\end{aligned}
$$

where

$\alpha$ is the path-loss exponent which is constant and generally set to be $1.6 \leq \alpha \leq 6 . d\left(s_{i}, r_{i}\right), d\left(s_{j}, r_{i}\right)$ and $d\left(c U E_{k}, r_{i}\right)$ are the distance between $\left(s_{i}, r_{i}\right),\left(s_{j}, r_{i}\right)$ and $\left(c U E_{k}, r_{i}\right)$ pair respectively. Equation (1) can be expressed in terms of distance as shown in equation (2).

$$
\operatorname{SINR}\left(r_{i}\right)=\frac{P\left(s_{i}\right) d\left(s_{i}, r_{i}\right)^{-\alpha}}{N+I+\operatorname{Inf}}
$$

where

$$
\begin{aligned}
\text { Inf } & =\sum_{c U E_{k}} P\left(c U E_{k}\right) d\left(c U E_{k}, r_{i}\right)^{-\alpha} \\
& +\sum_{j \neq i} P\left(s_{j}\right) d\left(s_{j}, r_{i}\right)^{-\alpha}
\end{aligned}
$$


The achievable data rate with co-channel interference, i.e., when channel is shared, is expressed as shown in equation (3) where $W$ is the channel bandwidth. Hereafter without loss of generality and for simplicity, we assume that $W=1$.

$$
R\left(r_{i}\right)=W \log _{2}\left(1+\frac{P\left(s_{i}\right) d\left(s_{i}, r_{i}\right)^{-\alpha}}{N+I+\operatorname{Inf}}\right)
$$

where

$$
\begin{aligned}
\text { Inf } & =\sum_{c U E_{k}} P\left(c U E_{k}\right) d\left(c U E_{k}, r_{i}\right)^{-\alpha} \\
& +\sum_{j \neq i} P\left(s_{j}\right) d\left(s_{j}, r_{i}\right)^{-\alpha}
\end{aligned}
$$

When the receiver is joining as a cUE, i.e., receiving content from the BS in cellular link, it experiences interference from D2D pair, which it will share the channel with, as shown in Figure 2. The SINR it experiences can be expressed as shown in equation (4) and the achievable data rate can be expressed as shown in equation (5).

$$
\begin{aligned}
& \operatorname{SINR}\left(c U E r_{i}\right)=\frac{P(e N B) d\left(e N B, c U E r_{i}\right)^{-\alpha}}{N+I+\sum_{j \neq i} P\left(s_{j}\right) d\left(s_{j}, c U E r_{i}\right)^{-\alpha}} \\
& R\left(c U E r_{i}\right)=W \log _{2}\left(1+\frac{P(e N B) d\left(e N B, c U E r_{i}\right)^{-\alpha}}{N+I+I n f}\right)
\end{aligned}
$$

where

$$
\operatorname{Inf}=\sum_{j \neq i} P\left(s_{j}\right) d\left(s_{j}, c U E r_{i}\right)^{-\alpha}
$$

where

$$
P(e N B) \quad \text { is transmit power of } e N B \text {. }
$$

$d\left(e N B, c U E r_{i}\right)$ is the distance between $e N B$ and $c U E r_{i}$.

$$
\begin{array}{ll}
P\left(s_{j}\right) & \text { is transmit power of } s_{j} . \\
d\left(s_{j}, c U E r_{i}\right) & \text { is the distance between } s_{j} \text { and } c U E r_{i} .
\end{array}
$$

Figure 2 Interference when receiving content in cellular link

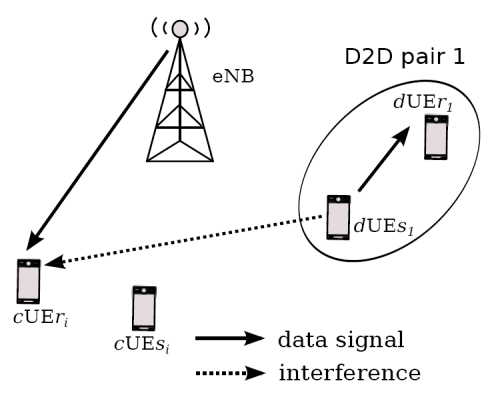

\subsection{Reward from $B S$}

When UEs do not have any benefit of taking part in D2D communication, i.e., they do not profit anything in terms of data transmission rate in our case, they expect reward from $\mathrm{BS}$ for receiving content in D2D link. The reward may be in the form of free data, coupons or other pre-scheduled high data rate period, etc. Similar to UEs, which look for their own profit for taking part in D2D communication, BS also does not offer rewards unless it has some benefit on doing so or it wants to fulfil its some pre-set criterion.

- $\quad B S$ reward criterion: Let $T_{\alpha}$ be the throughput BS wants to achieve and $T_{c}$ be the current throughput of BS. For given fixed $T_{\alpha}$ and $T_{c}$, if $T_{\alpha}>T_{c}$, BS offers reward to UEs for taking part in D2D communication.

- Reward function: Let $f_{r}\left(R_{i}\right)$ be the reward offered to $U E_{i}$ whose data rate is $R_{i}$ then $f_{r}\left(R_{i}\right)$ is defined as shown below in equation (6).

$$
f_{r}\left(R_{i}\right)=\frac{R_{i}+T_{c}}{T_{\alpha}}
$$

As $R_{i}$ increases, the value of $f_{r}\left(R_{i}\right)$ increases, i.e., $U E_{i}$ which offers maximum $R_{i}$ is rewarded highly. This is understandable because $U E_{i}$ which offers more $R_{i}$ consumes its resources more such as transmit power. The maximum achievable reward is when $\frac{R_{i}+T_{c}}{T_{\alpha}}=1$, after which BS may or may not increase/offer the reward. When BS increases the $T_{\alpha}$, it also has to increase the reward. It is obvious that, for given $T_{c}, R_{i}$ is linearly dependent to $T_{\alpha}$ and $f_{r}\left(R_{i}\right)$ is proportional to $R_{i}$.

\subsection{Implementation of D2D communication establishment}

When the BS (eNB) receives request for content from a new $\mathrm{UE}$ (rUE), it searches for a content provider within the D2D communication range. If there is one, it finds the locations of the requesting rUE and the content sender/provider sUE. The BS then calculates the achievable data rate in D2D communication mode, $\left(R\left(r_{i}\right)\right)$, and cellular communication mode, $\left(R\left(c U E r_{i}\right)\right)$, for the rUE. It sends $R\left(r_{i}\right), R\left(c U E r_{i}\right)$ and the locations of the rUE and the sUE. Upon receiving the above information, the rUE recalculates $R\left(r_{i}\right)$ and $R\left(c U E r_{i}\right)$ for confirmation. If $R\left(r_{i}\right)$ is higher than $R\left(c U E r_{i}\right)$, it replies that it will take part in D2D communication mode without reward, if not it will reply asking for reward for taking part in $\mathrm{D} 2 \mathrm{D}$ communication. When the $\mathrm{BS}$ receives the reply without reward it establishes D2D communication mode between the content receiver/requester and the content sender. If it receives reply with reward, it offers the reward which the content requester may or may not accept it. If the content requester accepts the reward, the D2D communication mode is established, otherwise the BS serves the content requester in cellular mode. The algorithms that are executed at the BS and at a rUE are shown in Algorithm 1 and Algorithm 2 respectively. 


\section{Simulation results and analysis}

In our simulation, we put 1,000 UEs randomly in a circular area of 500 metre radius with eNB in the centre. We choose 40 cUEs randomly as active UEs, i.e., spectrum they are using will be shared by dUEs. Other parameters used in simulation are shown in Table 1. One non-active UE is chosen randomly as content requester and the other one which is in $\mathrm{D} 2 \mathrm{D}$ communication range is chosen randomly as content provider, i.e., to from D2D pair. The simulation is executed 1,000 times in each scenario to find the percentage of content requesters asking for reward to take part in D2D communications. We also show the minimum average reward a UE ask to take part in D2D communication. The reward is presented as unit not as a particular value, e.g., coupon, data plan, etc. Furthermore, we show the ratio of achievable transmission rate of D2D link without reward and with reward. The value of the ratio tells us if D2D communication can be promoted without reward also.

Algorithm 1 Execution at eNB When rUEs Requesting for Contents

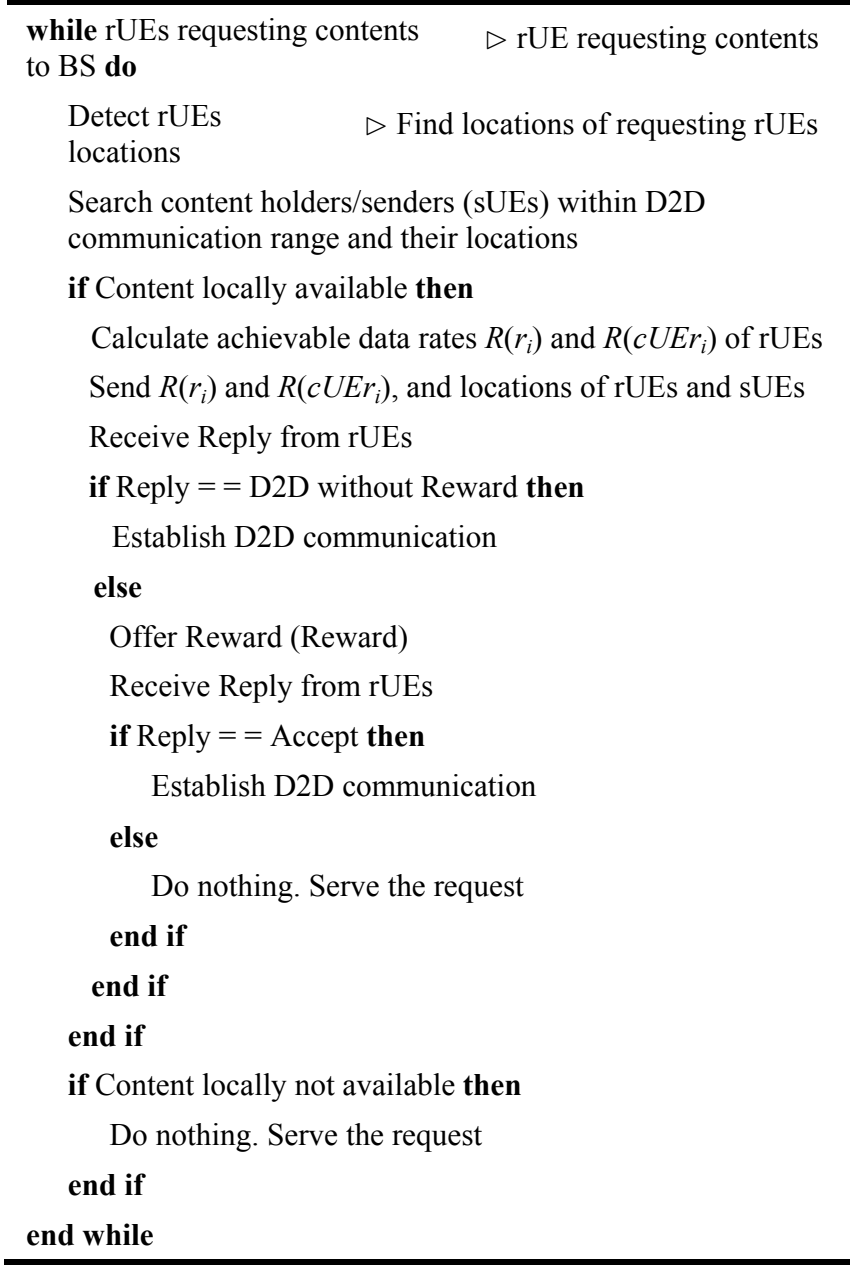

Algorithm 2 Execution at rUEs

Send Request for content to BS

Wait response from $\mathrm{BS}$

Receive $R\left(r_{i}\right)$ and $\mathrm{R}\left(c U E r_{i}\right)$, and locations of rUEs and sUEs from BS

Calculate achievable data rate $R\left(r_{i}\right)$ and $R\left(c U E r_{i}\right)$

if $R\left(r_{i}\right)>R\left(c U E r_{i}\right)$ then

Reply D2D communication mode without Reward else

Reply D2D with Reward Request

Receive Reward Offer from BS

if Reward Offer $\geq$ Reward Request then

Send Accept

else

Send Reject

end if

end if

Figure 3 shows that as the number of D2D pairs that share the channel increases, the number of UEs asking for reward for taking part in D2D communication increases. When the number of D2D pairs increases SINR decreases as there will be many pairs sharing the same channel and more interference at the D2D pairs causing less achievable data rate in D2D communication link than in cellular link. UEs will prefer to be served by BS unless there is reward for taking part in D2D mode.

In Figure 4, we show the effect of D2D communication distance. As the distance increases SINR decreases as the received signal power at the $\mathrm{D} 2 \mathrm{D}$ receiver decreases resulting decrease in achievable data rate in D2D link compared to cellular link. Thus the percentage of D2D pair asking for reward increases as the D2D distance increases. It is desirable to have D2D transmission distance shorter to encourage users to take part in D2D communication without reward.

Table 1 Simulation/numerical parameters

\begin{tabular}{lc}
\hline Parameters & Values \\
\hline Cellular area radius & $500 \mathrm{~m}$ \\
Maximum D2D distance & $30 \mathrm{~m}$ \\
Number of active cellular UE & 40 \\
Noise spectral density & $-174 \mathrm{dBm} / \mathrm{Hz}$ \\
Maximum eNodeB transmit power & $40 \mathrm{~W}$ \\
Maximum UE transmit power & $200 \mathrm{~mW}$ \\
Path-loss exponent $\alpha$ between eNodeB to UE & 3.5 \\
Path-loss exponent $\alpha$ between UE to UE & 4 \\
\hline
\end{tabular}


Figure 3 Percentage of UEs asking for rewards as number of D2D pair varies (see online version for colours)

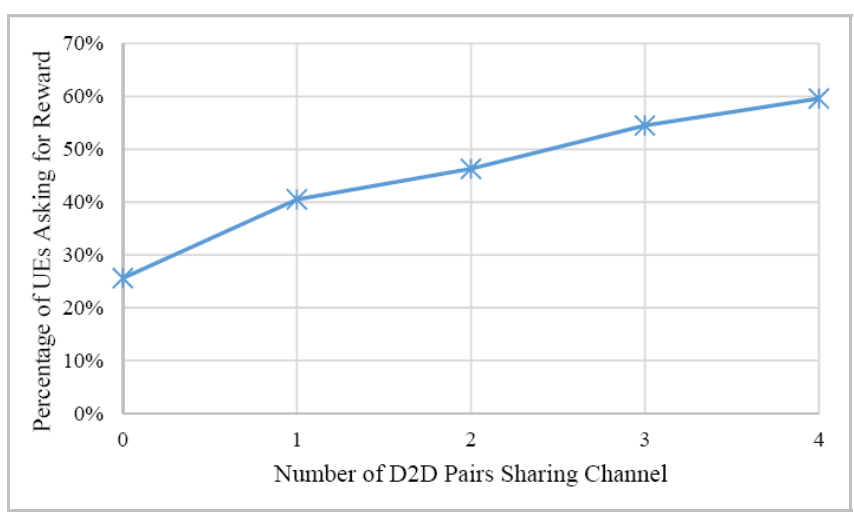

Figure 4 Percentage of UEs asking for reward as D2D distance varies (see online version for colours)

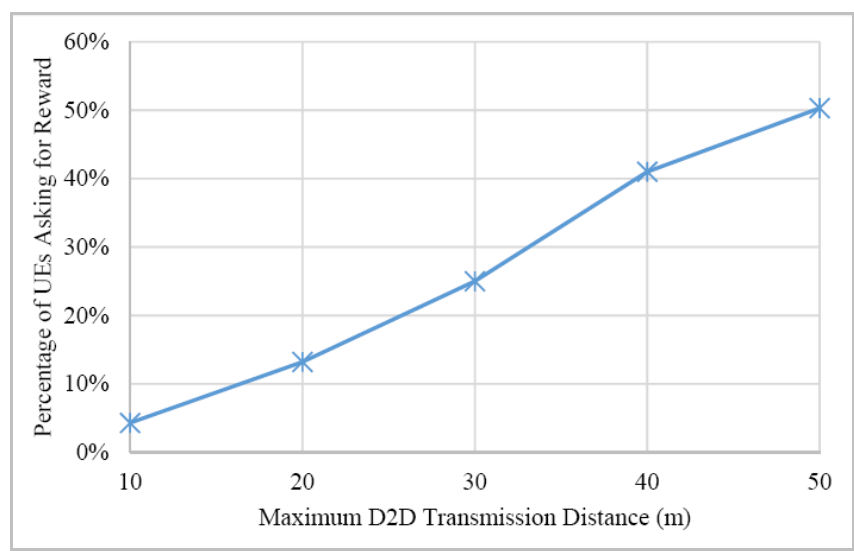

Figure 5 Percentage of UEs asking for reward as network size varies (see online version for colours)

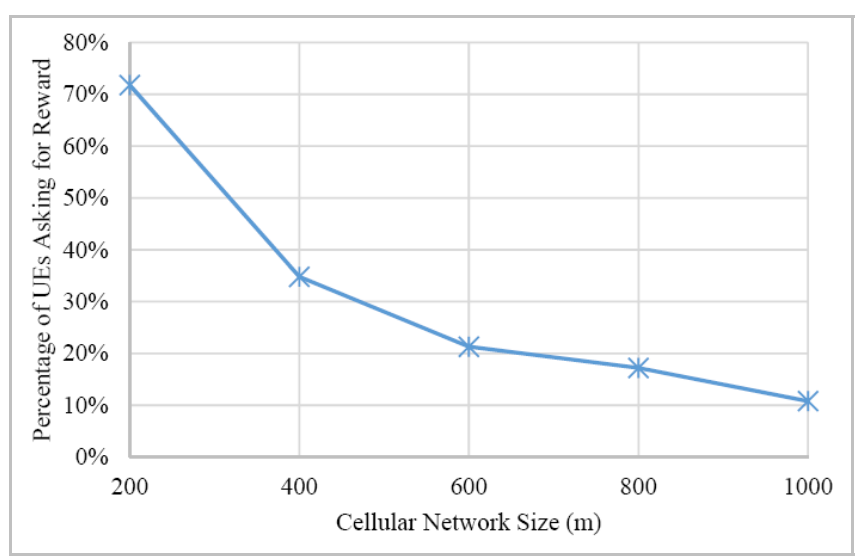

Figure 5 shows how the number of UEs asking for reward to take part in D2D communication mode changes as the network size varies. As the network size increases the interference to D2D pair decreases resulting higher SINR, i.e., some D2D pairs will have higher achievable data rate in D2D link than cellular link thus number of D2D pairs asking for reward to take part in D2D communication decreases as the network size increases. In a larger network size, the density of given active cUEs will be less, i.e., less interference to $\mathrm{D} 2 \mathrm{D}$ pairs. It is a preferable scenario to promote D2D communication without requiring reward for users to take part in D2D communication.

Figure 6 Average requested reward as number of D2D pairs varies (see online version for colours)

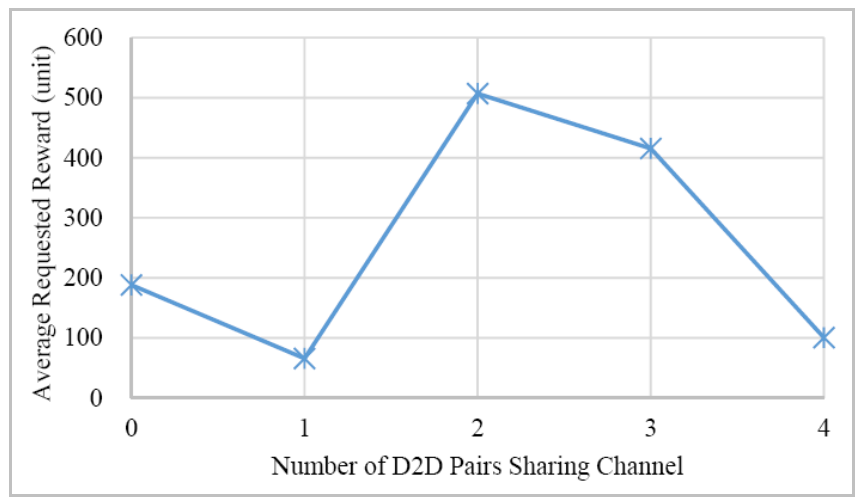

Figure 7 Average requested reward as D2D distance varies (see online version for colours)

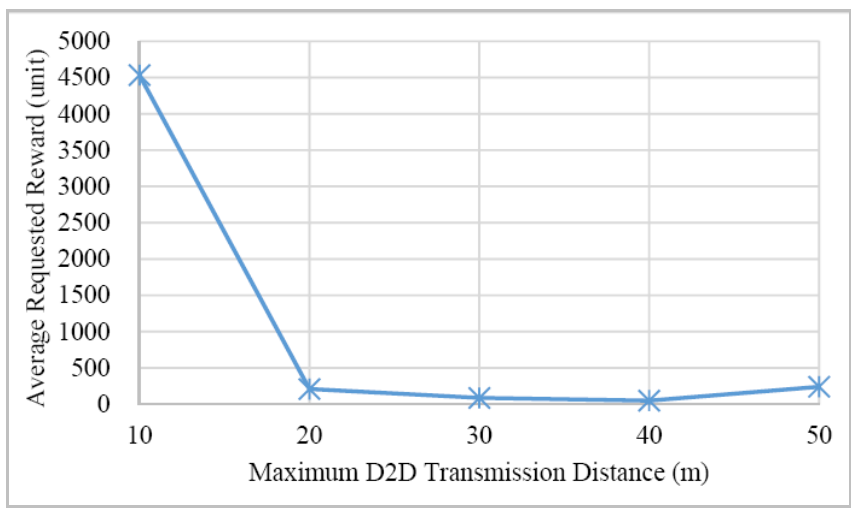

Figure 8 Average requested reward as network size varies (see online version for colours)

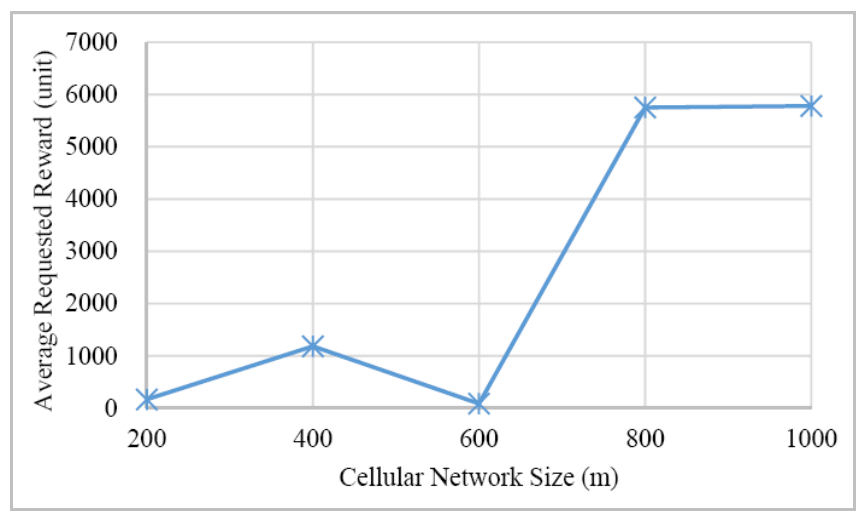

Even though there are some D2D pairs which are willing to take part in D2D communication without rewards, there are still some D2D pairs which will ask for reward for taking part in D2D communication as their achievable data transmission rate in $\mathrm{D} 2 \mathrm{D}$ mode is less than in cellular mode. In Figures 6, 7 and 8 we show the minimum average reward UEs ask for taking part in D2D communication mode. As 
we can see from the figures, the reward asked by UEs does not exhibit any particular pattern in terms of D2D distance, network size or number of D2D pairs sharing the channel. It depends upon the network throughput at the time of UEs requesting content and the achievable data rate in D2D link and in cellular link.

Figure 9 Ratio of achievable transmission rate of D2D links without and with awards as number of D2D pairs varies (see online version for colours)

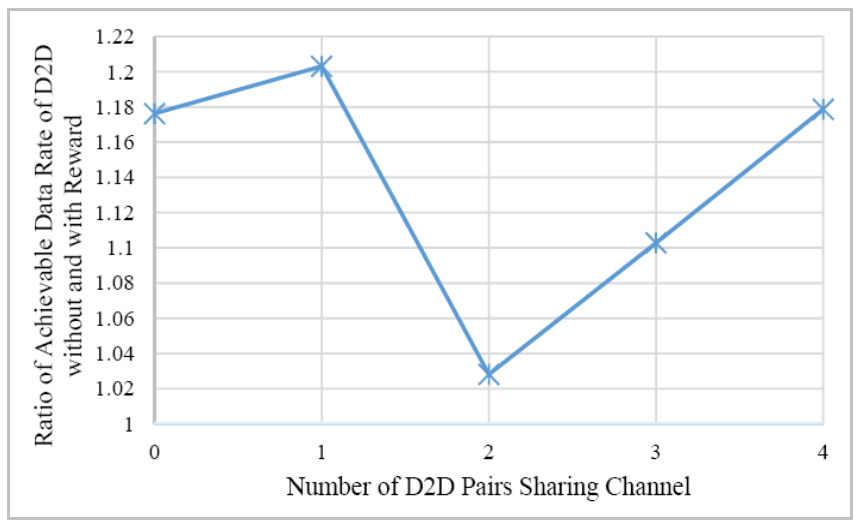

Figure 10 Ratio of achievable transmission rate of D2D links without and with awards as D2D distance varies (see online version for colours)

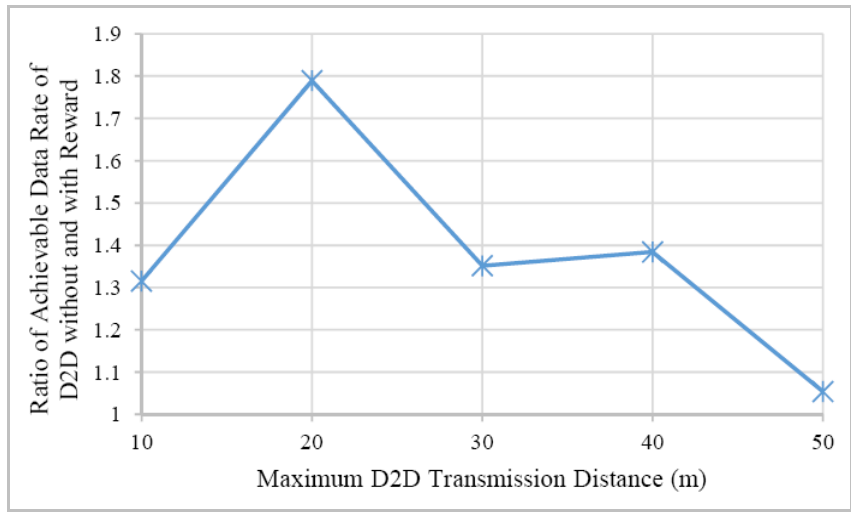

Figure 11 Ratio of achievable transmission rate of D2D links without and with awards as network size varies (see online version for colours)

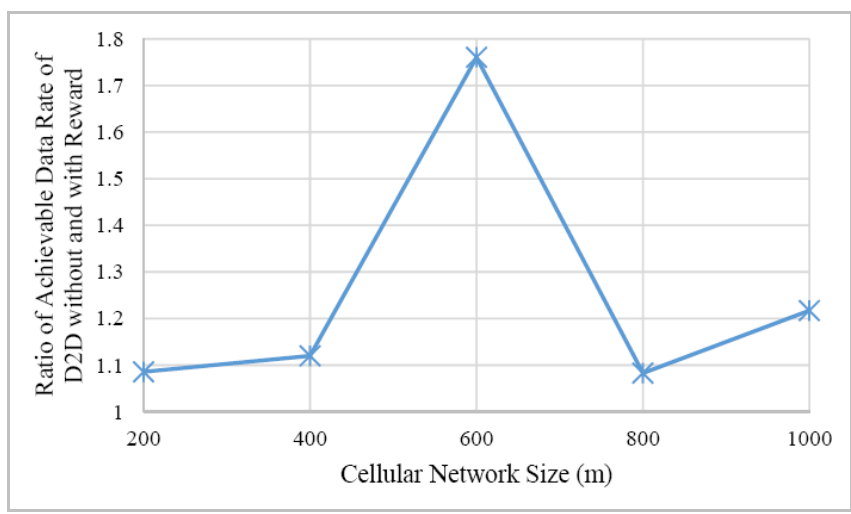

Figures 9, 10 and 11 show the ratio of average achievable data transmission rate of D2D pairs without and with reward. Although the ratio does not exhibit any particular pattern with respect to the number of D2D pairs sharing channel, D2D distance or cellular size, it is above 1 in all scenarios. From the results we can see that average achievable data transmission rate in D2D link in different scenarios and random location of D2D pairs is higher than cellular link encouraging users to take part in D2D communications without rewards.

\section{Conclusions}

In this paper, we presented a mechanism to show the achievable data rates in D2D link and cellular link to UEs when they request BS for content. When the achievable data rate in D2D link is less than in cellular link UEs will ask for reward for taking part in D2D communication otherwise they may willfully take part in D2D communication without reward. We show the percentage of UEs asking for reward by simulation by varying network size, number of D2D pairs and D2D distance. From the simulation we find that all UEs do not ask for reward if they know that they benefit more from D2D link than cellular link. We also show that the reward asked does not depend upon the above parameters. Furthermore, we presented the ratio of average achievable data rate in D2D link without and with reward. From the simulation results we can see that users may take part in D2D communication without reward if they are informed and if they can confirm also that the achievable data rate in D2D link is higher than cellular link. Although it is important to formulate how reward will be awarded to D2D pairs as shown in related works, it is equally important to inform users about achievable data rate in D2D and cellular links in order to promote D2D communications in cellular networks.

\section{References}

3GPP (n.d.) Specification Releases [online] http://www.3gpp.org/ specifications/releases (accessed 28 April 2017).

Ansari, R.I., Hassan, S.A. and Chrysostomou, C. (2016) 'Energy efficient relay selection in multi-hop D2D networks', in 2016 International Wireless Communications and Mobile Computing Conference (IWCMC), pp.620-625.

Asadi, A., Wang, Q. and Mancuso, V. (2014) 'A survey on device-to-device communication in cellular networks', IEEE Communications Surveys Tutorials, Vol. 16, No. 4, pp.1801-1819.

Chen, Y., He, S., Hou, F., Shi, Z. and Chen, J. (2017) 'Promoting device-to-device communication in cellular networks by contract-basesd incentive mechanisms', IEEE Network, Vol. 31, No. 3, pp.14-20. 
Cisco (2017) Cisco Visual Networking Index: Global Mobile Data Traffic Forecast Update, 2016-2021 White Paper, March [online] http://www.cisco.com/c/en/us/solutions/collateral/ service-provider/visual-networking-index-vni/mobile-whitepaper-c11-520862.html (accessed 28 April 2017).

Datsika, E., Antonopoulos, A., Zorba, N. and Verikoukis, C. (2016) 'Green cooperative device-to-device communication: a social-aware perspective', IEEE Access, Vol. 4, pp.3697-3707.

Doppler, K., Rinne, M., Wijting, C., Ribeiro, C.B. and Hugl, K. (2009) 'Device-to-device communication as an underlay to LTE-advanced networks', IEEE Communications Magazine, Vol. 47, No. 12, pp.42-49.

Ghosh, A., Ratasuk, R., Mondal, B., Mangalvedhe, N. and Thomas, T. (2010) 'LTE-advanced: next-generation wireless broadband technology [invited paper]', IEEE Wireless Communications, Vol. 17, No. 3, pp.10-22.

Li, P. and Guo, S. (2015) 'Incentive mechanisms for device-to-device communications', IEEE Network, Vol. 29, No. 4, pp.75-79.

Lin, X., Andrews, J.G. and Ghosh, A. (2014) 'Spectrum sharing for device-to-device communication in cellular networks', IEEE Transactions on Wireless Communications, Vol. 13, No. 12, pp.6727-6740.
Xiao, Y., Chen, K.C., Yuen, C. and DaSilva, L.A. (2014) 'Spectrum sharing for device-to-device communications in cellular networks: a game theoretic approach', in 2014 IEEE International Symposium on Dynamic Spectrum Access Networks (DYSPAN), pp.60-71.

Ye, Q., Al-Shalash, M., Caramanis, C. and Andrews, J.G. (2014) 'Resource optimization in device-to-device cellular systems using time-frequency hopping', IEEE Transactions on Wireless Communications, Vol. 13, No. 10, pp.5467-5480.

Yu, C.H., Doppler, K., Ribeiro, C.B. and Tirkkonen, O. (2011) 'Resource sharing optimization for device-to-device communication underlaying cellular networks', IEEE Transactions on Wireless Communications, Vol. 10, No. 8, pp.2752-2763.

Zhang, Y., Song, L., Saad, W., Dawy, Z. and Han, Z. (2015) 'Contract-based incentive mechanisms for device-to-device communications in cellular networks', IEEE Journal on Selected Areas in Communications, Vol. 33, No. 10, pp.2144-2155.

Zulhasnine, M., Huang, C. and Srinivasan, A. (2010) 'Efficient resource allocation for device-to-device communication underlaying LTE network', in 2010 IEEE 6th International Conference on Wireless and Mobile Computing, Networking and Communications, pp.368-375. 